INRA Prod. Anim.

2014, 27 (1), 17-30

\title{
Quelles perspectives pour les filières laitières de montagne après la suppression des quotas laitiers? Une approche en termes de régime de concurrence
}

\author{
M. DERVILLE' ${ }^{1}$, G. ALLAIRE' \\ 1 UMR Dynamiques Rurales, ENFA BP 22687, F-31326 Castanet-Tolosan, France \\ 2 INRA, US0685 Observatoire des Programmes Communautaires de Développement Rural, \\ F-31326 Castanet-Tolosan, France \\ Courriel : marie.derville@educagri.fr
}

La décision européenne de suppression des quotas laitiers dans un contexte de globalisation et de concentration des firmes interroge les évolutions structurelles et spatiales de la production. Les systèmes de production de montagne, dont l'ancrage reposait principalement sur la gestion départementalisée des quotas laitiers, semblent particulièrement fragilisés. La structuration de l'action collective pour construire une offre de lait adaptée à la demande et l'accompagnement des pouvoirs publics sont des enjeux forts pour l'avenir des filières laitières de montagne.

La production laitière de montagne occupe une place non négligeable au niveau national : 17958 producteurs, 3,5 milliards de litres de lait produits (campagne $2009 / 2010$ ) soit $22 \%$ des producteurs et $15 \%$ des volumes nationaux (Dervillé 2012, données FAM 2009 -Annexe 1-). C'est aussi 40\% des entreprises laitières françaises et $20 \%$ des fabrications fromagères françaises (id., données EAL 2006 -Annexe 1-). Toutefois, du fait des contraintes pédoclimatiques, de la pente et de l'éloignement, la production de montagne est affectée de surcoûts de production évalués à $30 \%$ par rapport à la plaine (CNASEA 2008). En outre, la densité laitière de montagne est deux à trois fois inférieure à celle des zones de plaine spécialisées. Les entreprises laitières, qu'elles soient des filiales de groupes nationaux ou des Petites et Moyennes Entreprises (PME), doivent ainsi faire face à des surcoûts de collecte du lait (en moyenne $15 € / 1000$ $\mathrm{L}$ de plus qu'en plaine) et de logistique importants (CNIEL 2011) ${ }^{1}$. Ainsi, les systèmes de production laitiers de montagne sont peu avantagés au regard de la concurrence sur les coûts de production.

Les quotas laitiers mis en place en 1984 avaient permis de protéger ces systèmes laitiers. Tout en réduisant le coût de la stabilisation du marché européen (mécanismes d'intervention moins sollicités du fait du rationnement), ce dispositif de coordination multi-acteurs et multi-niveaux a en effet permis de limiter la concurrence entre producteurs et entre territoires (Dervillé et al 2012). La concentration de la production dans le Grand-Ouest de la France a notamment été freinée (Chatellier et Delattre 2003, Kroll et al 2010). Cependant, en 2008, la Commission Européenne a pris la décision de libéraliser les marchés laitiers : poursuite de la baisse de l'intervention et augmentation progressive des quotas jusqu'à leur suppression en 2015. De ce fait, les systèmes laitiers de montagne se retrouvent plus directement en compétition avec des systèmes de plaine, systèmes qui sont amenés à accroître les volumes de production et à renforcer leur compétitivité-coût (Lelyon et al 2012). Plus flexibles, ils sont également plus aptes à faire face à la volatilité croissante des cours, comme en témoigne leur capacité à bénéficier des hausses de prix en 2007-2008 par un accroissement de la production alors que les systèmes de montagne n'ont subi que la baisse de 2008-2009 (Institut de l'Elevage 2009a). La pérennité des systèmes de montagne s'en trouve menacée.
Les systèmes de montagne bénéficient pourtant de soutiens spécifiques, dans le cadre du second pilier de la Politique Agricole Commune (politique de la montagne et primes herbagères), qui peuvent renforcer leur compétitivité. Ces soutiens n'ont longtemps fait que compenser les inégalités des soutiens versés dans le cadre du premier pilier (Dervillé et al 2012). Avec la création d'une prime à l'herbe productive, le renforcement des Indemnités Compensatoires de Handicap Naturel (ICHN) et la création d'une aide au lait de montagne, la réforme du bilan de santé de 2008 s'est toutefois accompagnée d'une hausse d'environ $25 \%$ du revenu des éleveurs laitiers de montagne (Chatellier et Guyomard 2009). Les négociations en cours sur la mise en œuvre de la PAC post-2015 constituent donc un enjeu pour la viabilité des systèmes laitiers de montagne. Mais, des mesures de soutien direct aux exploitations ne peuvent être le ressort du maintien de la production laitière dans les régions de montagne que si ces mesures sont associées à une stratégie de filière, comme l'ont montré plusieurs études (Ricard et Rieutort 1995, Chatellier et Delattre 2006).

Les démarches de segmentation des marchés, basées sur une différenciation territorialisée des systèmes productifs,

${ }^{1}$ Déclaration de la commission de montagne du Centre National Interprofessionnel de l'Économie Laitière. 
peuvent contribuer à séparer les espaces de concurrence et favoriser l'ancrage territorial de la production en montagne après les quotas. En effet, la création d'une demande particulière est à l'origine d'une certaine rareté qui peut générer une rente de qualité territoriale (Pecqueur 2001) et renforcer la compétitivité des systèmes productifs concernés. Il faut néanmoins s'interroger sur la pérennité de ces stratégies de différenciation alors que des possibilités d'accroissement de la production et de concentration de l'offre sont offertes aux producteurs. Dans quelle mesure les organismes de défense et de gestion de ces produits de qualité spécifique vont-ils pouvoir continuer à contrôler la dynamique de ces filières ? En outre, seules $30 \%$ des livraisons de montagne sont valorisées en Appellation d'Origine Protégée (AOP). Les $70 \%$ restant sont valorisés dans des filières génériques les mettant directement en concurrence avec les livraisons de plaine. Est-ce que les producteurs et les transformateurs de ces territoires auront la capacité d'initier de nouvelles stratégies de segmentation des marchés? Le renforcement du mode de consommation éthique et des préoccupations nutritionnelles, malgré la crise économique, constitue une incitation en ce sens. Reste à savoir si les systèmes laitiers de montagne sauront renforcer et faire valoir leurs spécificités. Parviendront-ils à s'appuyer sur les opportunités offertes par le Paquet Lait adopté en mars 2012 (contractualisation et organisations de producteurs) pour élaborer de nouveaux dispositifs de stabilisation des marchés?

Pour évaluer les potentialités de différenciation des systèmes productifs et des marchés et les potentialités d'ancrage territorial qui en découlent, nous avons cherché à analyser, dans un même cadre, les dynamiques méso-économiques (à partir d'études de cas à l'échelle départementale) et leurs interactions avec les transformations de niveaux macro-économique et micro-économique. Dans une première partie, les fondements théoriques de ce cadre d'analyse $\mathrm{du}$ fonctionnement des marchés sont explicités. Le concept de régime de concurrence, qui rend compte de la position d'un système productif régional dans la dynamique des marchés, y est défini. Dans une deuxième partie, l'hétérogénéité des ressources disponibles en montagne et son impact sur les dynamiques laitières sont mises en évidence. Dans une troisième partie, le cadre conceptuel proposé permet d'analyser, à partir de cas-types, les leviers et perspectives d'ancrage de la production à l'œuvre dans trois régimes de concurrence contrastés. La quatrième partie, en combinant les résultats des études de cas et de l'analyse statistique des dynamiques laitières, permet de caractériser et de cartographier les quatre principaux enjeux d'adaptation post-quotas, et de proposer des leviers potentiels de libération des capacités d'organisation collective.

Encadré 1. Approche institutionnelle de la concurrence et de la compétitivité.

Concurrence : compétition entre entreprises et entre ensembles d'entreprises produisant les mêmes biens pour accéder au marché.

Compétitivité-coût : capacité d'une entreprise à faire face à la concurrence par les prix.

Compétitivité hors-prix ou hors-coût : capacité à se dégager de la concurrence sur les prix par la différenciation des marchés. Elle désigne les facteurs autres que les prix et les coûts qui contribuent à la compétitivité, comme la qualité, l'innovation, l'image de marque, les services associés (logistique, service après vente...).

Régime de concurrence : arrangement institutionnel stabilisé dans des dispositifs de coordination à l'origine du contrôle des formes de la concurrence sur un marché ou un ensemble de marchés, qui réduit les incertitudes et permet l'anticipation des acteurs économiques. Ces dispositifs de coordination recouvrent des instruments de politique publique, des structures de coordination entre participants aux marchés (professions, interprofessions, standards de qualité), des infrastructures, des stratégies et des dispositifs d'innovation, reposant sur et créant des ressources immatérielles communes. Un régime de concurrence articule des règles formelles (à différents niveaux d'organisation et de droits) et des règles informelles, influençant les conduites individuelles. Un régime de concurrence peut être défini à un niveau macro-économique (échelle nationale ou européenne dans le cas présent), mais aussi à un niveau méso-économique (échelle régionale de la différenciation des marchés). Dans le cadre d'un ordre institutionnel macro-économique, les innovations institutionnelles régionales marquent en effet les rapports de concurrence et peuvent transformer les conditions d'accès aux marchés des producteurs.

Espace de concurrence : segment de marché résultant de la différenciation spatiale et sectorielle d'un régime (macro-économique) de concurrence. Dans le sens utilisé ici : échelle de déploiement des dispositifs de coordination sous-tendant un régime régional de concurrence ; il est caractérisé par une séparation singulière entre domaines de coopération et domaines de concurrence, se traduisant par des conditions particulières d'accès au marché.

\section{1 / Le régime régional de concurrence : un modèle multi-niveaux et multi- acteurs du fonctionnement des marchés laitiers}

\section{1 / Une analyse institutionnelle du fonctionnement des marchés}

Pour expliciter les dynamiques sectorielles et territoriales de la production sans partir d'un modèle normatif, l'observation du fonctionnement des marchés a constitué le point de départ de l'analyse. En se référant au rôle des institutions dans la stabilisation des marchés, ce travail s'inscrit dans la lignée des travaux « régulationnistes » (Boyer 1995, Touzard 1995, Allaire 2002, Boyer 2003). Dans ce cadre, certains secteurs ou territoires, ou certaines configurations secteur/territoire, se caractérisent par des spécificités et régularités de fonctionnement reposant sur des dispositifs de coordination particuliers. La sociologie économique (e.g., Fligstein 1996, White et al 2008, Allaire 2010) considère que la recherche de stabilité des opérateurs économiques équilibre la recherche de profit. " Le but de l'action sur un marché donné est de créer et de maintenir des univers stables au sein et entre firmes, qui leur permettent de survivre » (Fligstein 1996). Cette quête de stabilité recouvre à la fois des processus économiques (répétition des comportements, coopération dans l'élaboration de normes de qualité, segmentation des marchés) et des processus politiques (sélection, dans différents collectifs, par l'expérience, de règles d'action collective visant à rendre compatibles les comportements individuels). Ainsi, la compétitivité des firmes ne résulte pas uniquement de stratégies individuelles rivales, mais repose aussi sur la mise en commun de ressources et l'élaboration d'accords de coopération visant à stabiliser les marchés, en amont ou en aval (encadré 1). La compétitivité des firmes, qui s'inscrivent dans des filières territorialisées, a une dimension collective et institutionnelle. Dans les phases de transition entre ce que nous appellerons des régimes de concurrence, les opérateurs de marchés font plus que défendre leur position contre la concurrence ; ils cherchent à faire advenir les modalités de contrôle de la concurrence qui les avantagent (Fligstein 1996). Le lobbying et la communication participent à la construction de la compétitivité, où s'additionnent et se confrontent stratégies individuelles et collectives. Les politiques publiques, en légitimant différentes façons d'entreprendre et de coopérer (Lascoumes et Le Gales 2004), participent également à la construction sociale des formes de la concurrence et de la compétitivité. Ainsi, 
la compétitivité des firmes et les rapports de concurrence/coopération sur un marché reposent sur un arrangement institutionnel stabilisé dans des dispositifs de coordination (politiques publiques, modèles d'entreprises, normes de qualité et structures de gouvernance) que l'on qualifie ici de régime de concurrence.

\section{2 / Le régime régional de concurrence : définition}

La compétitivité des firmes a aussi une dimension territoriale. «L'ancrage territorial de la firme résulte avant tout de la mise en œuvre d'une construction commune territorialisée, [...] d'un apprentissage collectif fondé sur la coproduction de ressources » (Perrat et Zimmerman 2003). Le territoire constitue alors un système de ressources allant au-delà des externalités d'agglomération. Une communauté d'acteurs, régie par un système d'institutions et caractérisée par des régularités de fonctionnement, apparaît. La différenciation de cet espace de concurrence n'est possible que si les structures de gouvernance mises en place sont autorisées par le contexte institutionnel (politique de la qualité et politique de la concurrence notamment). Les dispositifs de coordination se déploient à plusieurs échelles.

Les régimes régionaux de concurrence désignent l'arrangement institutionnel des dispositifs de coordination délimitant les stratégies de concurrence possibles localement. La différenciation spatiale des régimes de concurrence peut provenir d'une appropriation différenciée des dispositifs publics et d'une trajectoire régionale d'innovation ayant favorisé la construction de ressources spécifiques (dépendance du sentier). Ainsi, selon les régions, les modèles d'entreprise et les marchés, les domaines de coopération (stratégie d'innovation, modèle de production, construction d'une réputation) et les domaines de concurrence entre opérateurs de marché sont distincts. Les opérateurs régionaux, agriculteurs compris, disposent ainsi de conditions particulières d'accès au marché. Les modalités de la restructuration du secteur sont donc spatialement différenciées.

\section{3 / Le régime régional de con- currence : le bien commun des opérateurs de marché}

De l'analyse des systèmes régionaux de production en termes de régimes de concurrence, il ressort que la différenciation de ces régimes résulte de la mise en commun, plus ou moins effective et efficace, de ressources dans deux domaines : le développement d'une capacité d'innovation et la construction d'une réputation collective (Dervillé 2012, tableau 1). Les ressources mises en commun peuvent être matérielles (centre de recherche, organisme de contrôle...) ou immatérielles (valeurs professionnelles, culture de métier, dispositifs de coordination, standards de qualité, cahiers des charges, statuts et droits...). Ces ressources peuvent être génériques (droits à produire à valeur faiblement dépendante du contexte territorial) ou spécifiques (rentes de qualité territoriales créées dans les filières AOP ou issues de processus localisés d'apprentissage et donc peu diffusables).

La notion de système de ressources communes développée par E. Ostrom (Ostrom et Schlager 1992, Ostrom 2000, Hess et Ostrom 2003) peut être étendue à la capacité collective à différencier et à stabiliser les marchés. Les stratégies régionales d'innovation, les modèles d'entreprises, les dispositifs de coopération sont en effet des règles d'action collective, qu'ont élaborées les acteurs de marché et auxquelles ils se soumettent, dans l'objectif de pouvoir bénéficier d'un environnement stabilisé. Ces règles formelles ou informelles (pratiques) conditionnent l'usage des ressources communes (management) et délimitent une communauté de bénéficiaires (exclusion des opérateurs qui ne se conformeraient pas aux règles collectives). Ces règles, en légitimant différentes actions relatives aux ressources, sont à l'origine de droits et de devoirs pour les membres de la communauté. Ces droits sur les ressources sont divers.
Ostrom et Schlager (1992) en distinguent cinq : l'accès, l'usage, le management, l'exclusion et l'aliénation. Tous les membres de la communauté (ici tous les participants au marché) n'ont pas accès aux mêmes droits. Dans le cas des régimes de concurrence, le système de ressources est constitué des dispositifs de coordination et des structures de coopération qui soutiennent les processus d'innovation et de création de valeur. Il n'est pas en soi appropriable et aliénable ; il repose sur des structures collectives (organisations professionnelles et interprofessionnelles) et implique l'acteur public. Les unités de ressources appropriables individuellement par les agriculteurs et les transformateurs sont notamment des compétences techniques ou organisationnelles, l'usage d'un nom, l'accès à des prix stables.

$\mathrm{Au}$ final, le concept de régime de concurrence et l'analyse en termes de système de ressources communes permettent de qualifier les modalités d'accès aux marchés des producteurs et d'identifier les acteurs qui participent à leur élaboration. Les régimes régionaux de concurrence, pour la présente recherche, ont été caractérisés par deux voies : une analyse statistique des processus de restructuration de la production laitière et une analyse qualitative des jeux d'acteurs, conduites chacune à différentes échelles (encadré 2). L'objectif est d'apporter un regard novateur sur les processus socioéconomiques et politiques à l'origine de la différenciation des marchés et des espaces de concurrence.

\section{2 / Des ressources hétérogè- nes à l'origine de dynamiques laitières contrastées}

\section{1 / Une répartition hétérogène des volumes de lait et des AOP}

La production laitière de montagne n'est pas répartie de façon homogène sur les cinq massifs (carte 1). Le Massif Central rassemble en 2009 près de 12000 producteurs pour un quota de

Tableau 1. Le régime régional de concurrence : un système de deux biens communs (d'après Dervillé 2012).

\begin{tabular}{|l|l|l|}
\hline & Capacité d'innovation & Réputation collective \\
\hline $\begin{array}{l}\text { Ressources mises en } \\
\text { commun }\end{array}$ & $\begin{array}{l}\text { Politique des structures } \\
\text { Structure de gouvernance (Syndicat, Commission } \\
\text { Départementale d'Orientation Agricole) } \\
\text { Programme de recherche et d'appui technique }\end{array}$ & $\begin{array}{l}\text { Politique de la qualité } \\
\text { Structures de gouvernance (Organisme } \\
\text { de Défense et de Gestion, cahier des } \\
\text { charges...) }\end{array}$ \\
\hline $\begin{array}{l}\text { Ressources appropriables } \\
\text { individuellement }\end{array}$ & $\begin{array}{l}\text { Compétences } \\
\text { Solutions techniques }\end{array}$ & $\begin{array}{l}\text { Nom } \\
\text { Prime de marché } \\
\text { Stabilité des prix }\end{array}$ \\
\hline
\end{tabular}


Carte 1. Densité des exploitations laitières en 2009 avec les limites des départements et des massifs (Vosges, Jura, Alpes, Massif central, Pyrénées) (Dervillé 2012, données FranceAgriMer).

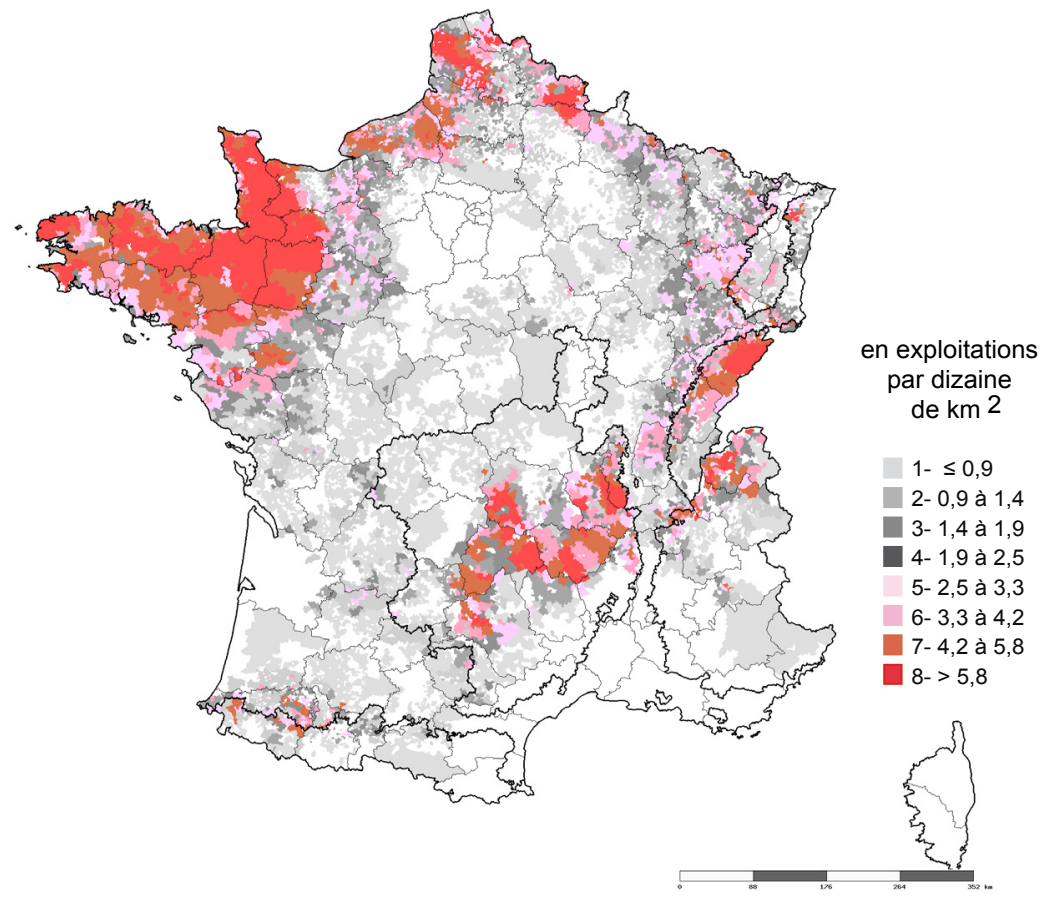

Carte 2. Taux de variation du nombre d'exploitations laitières sur 1999-2009 avec les limites des départements et des massifs (Données FranceAgriMer).

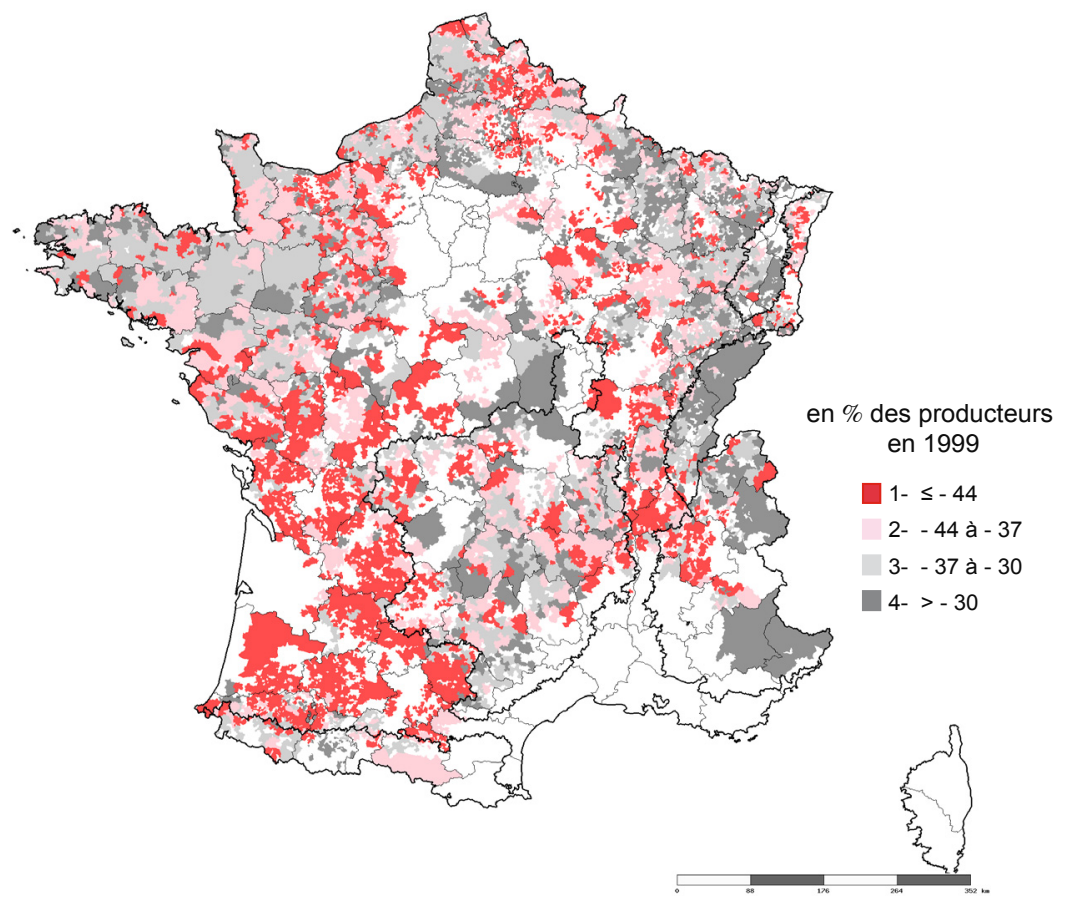

près de 2,3 milliards de litres, soit $66 \%$ des producteurs et $64 \%$ des droits à produire de montagne. Les massifs jurassien et alpin rassemblent chacun $15 \%$ de la production, pour respectivement 12 et $16 \%$ des producteurs. Les massifs des Vosges et des Pyrénées regroupent chacun $3 \%$ de la production laitière de montagne. res est importante en montagne, ce qui sécurise l'accès aux services techniques, favorise les échanges entre producteurs et préserve ainsi une « ambiance laitière » propice au maintien de la production (Chatellier et Pflimlin 2007, Paradis et al 2010, Dervillé 2012).

Les potentiels de valorisation sous AOP sont également hétérogènes. Dans le massif du Jura, $71 \%$ des droits à produire sont valorisables en $\mathrm{AOP}^{2}$. Pour la zone montagnarde du Doubs et du Jura, la totalité des volumes est prise en charge par ces filières de qualité spécifique. Dans les Alpes, les AOP permettent de valoriser $43 \%$ des droits à produire. Les AOP sont cependant concentrées sur les départements de Savoie et de Haute-Savoie, où $\mathrm{AOP}$ et Indications Géographiques de Provenance (IGP) valorisent la totalité du lait. Le reste des Alpes est par contre inséré dans des filières génériques. Dans le Massif Central, les AOP valorisent $20 \%$ des droits à produire; ce pourcentage atteint néanmoins $60 \%$ dans les départements du Cantal et du Puy de Dôme. L'AOP Munster valorise environ $18 \%$ des droits à produire de la montagne vosgienne. L'IGP Tome des Pyrénées valorise au plus $25 \%$ du lait de montagne.

Ainsi, les volumes produits en AOP ne permettent pas d'ancrer la production laitière sur l'ensemble des massifs. Surtout, la labellisation ne constitue pas une garantie de succès économique. Dans un même cadre juridique, la capacité collective à construire une réputation et à créer de la valeur est plus ou moins forte (Perrier-Cornet et Sylvander 2000, Vandecandelaere et al 2009, Dervillé 2012). Les droits effectifs des éleveurs sur les ressources spécifiques créées sont en effet variables.

En outre, le profil des entreprises laitières est contrasté. Les exploitations du Jura sont plus grandes et modernisées que celles du Massif Central qui ont investi plus tardivement (Institut de l'Elevage 2009a). Les quotas par exploitation sont supérieurs de $23 \%$ dans le Jura. Les différents massifs ne disposent pas non plus du même potentiel de transformation : à l'exception des Vosges, du massif du Jura et des Alpes du Nord, la montagne est dépendante des zones laitières de plaine voisines pour transformer son lait. En 2006, le tiers du lait de montagne (989 millions de litres) aurait été transformé hors montagne (Dervillé 2012, données EAL 2006). Si les établissements de montagne sont en moyenne de dimension plus faible qu'en plaine, Jura et Alpes du Nord se distinguent par une persistance de fromageries artisanales alors que la transformation est largement industrialisée sur les autres massifs.

\footnotetext{
${ }^{2}$ Les pourcentages ont été estimés en croisant les volumes AOP, les droits à produire géo-localisés et les taux de réalisation des quotas à l'échelle des départements.
} 
Carte 3. Part des formes sociétaires en 2009 (Données FranceAgriMer).

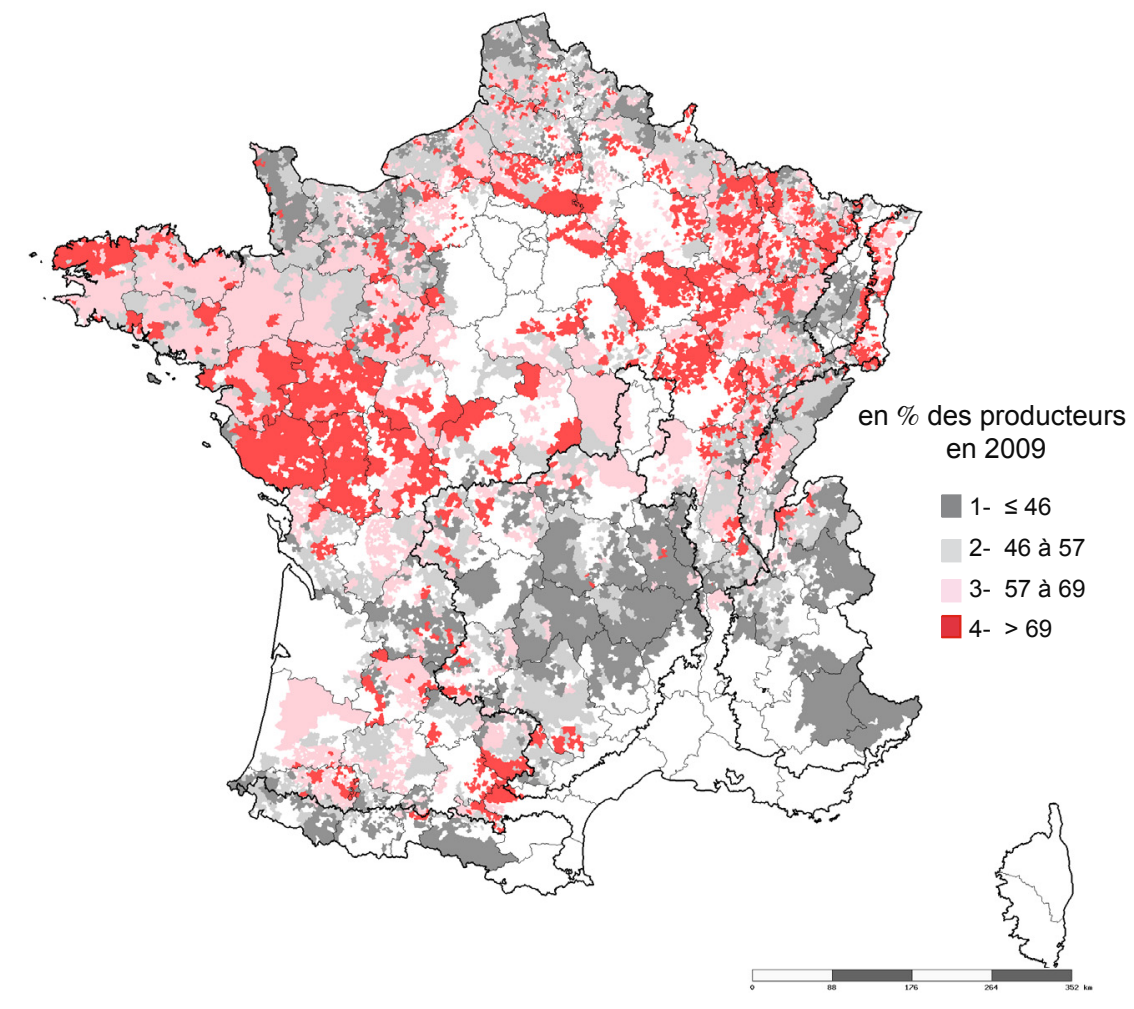

Ainsi densité laitière, modèles d'entreprise, distance aux sites de transformation, présence d'AOP et potentiel de valorisation permettent de différencier les territoires de montagne. Quel impact la présence de ces différentes ressources a-t-elle sur les conditions d'accès au marché des producteurs et sur les dynamiques de restructuration laitière ?

\section{2 / Diversité spatiale des méca- nismes de la restructuration lai- tière}

Le traitement de données exhaustives et géo-localisées sur les exploitations laitières permet de comparer les dynamiques laitières entre territoires de montagne et de plaine, ainsi qu'entre microterritoires au sein de l'ensemble montagne (encadré 2). Sur 1999-2009, la restructuration laitière s'est poursuivie avec une perte de $38 \%$ de producteurs. Cette restructuration s'est faite à volumes croissants à l'échelle de la France $(+3,6 \%)$ et avec un fort développement des formes sociétaires d'exploitation agricole (cartes 2 et 3 ).

La montagne se caractérise par une diminution légèrement plus faible du

Encadré 2. Méthodes de caractérisation des régimes de concurrence (Dervillé 2012).

\section{Analyse qualitative des stratégies d'acteurs}

L'analyse de la configuration des opérateurs sectoriels et territoriaux et de la transformation dans le temps des régimes régionaux de concurrence repose sur une analyse secondaire de la littérature et sur un travail d'enquêtes réalisé entre 2008 et 2011 auprès d'acteurs du marché. Une double série d'entretiens semi-directifs a également été conduite auprès de différents acteurs de la filière (opérateurs et acteurs institutionnels) à l'échelle nationale et au sein des trois départements d'étude retenus pour caractériser des enjeux d'adaptation à l'après-quotas (Cantal, Haute-Loire et Doubs). Le département, support de la mise en œuvre des stratégies modernisatrices de la politique de développement agricole depuis les années 1960, a été considéré comme point d'entrée de l'analyse des configurations productives.

\section{Rapprochement de bases de données géo-localisées et construction d'indicateurs individuels (exploitations, entreprises laitières) et territoriaux}

L'analyse statistique repose sur la mise en relation originale de dix bases de données géo-référencées à l'échelle communale, grâce à la plateforme informatique de l'Observatoire du Développement Rural (INRA de Toulouse) (Tableau 2). Cinq de ces bases correspondent à des données individuelles exhaustives relatives aux exploitations ou entreprises laitières.

Pour définir les stratégies individuelles des exploitations laitières entre 2004 et 2009, différentes variables individuelles (taille du quota, statut juridique de l'exploitation, pratique de la vente directe, contractualisation de mesures agro-environnementales...) ont été construites. En outre, l'environnement technique, économique, institutionnel des exploitations a été caractérisé, à différentes échelles, à l'aide de variables territoriales (système de production dominant sur la zone, degré d'intensification des systèmes, densité laitière, mix produit et taille des entreprises d'aval dans un rayon de $50 \mathrm{~km}$, poids local des aides du second pilier, présence d'AOP...).

\section{Appréhender les échelles de fonctionnement des marchés laitiers}

Différentes échelles d'analyse ont été considérées pour se rapprocher de territoires pertinents pour le fonctionnement du secteur. Deux types de territoires infra-départementaux ont été construits pour pouvoir appréhender les contrastes de la dynamique laitière :

- les micro-zones laitières sont des unités statistiques élaborées par le regroupement de communes laitières contiguës homogènes pour certaines caractéristiques. Sont intégrés ici les zonages Indemnités Compensatoires de Handicap Naturel (ICHN), AOP, Petite Région Agricole (PRA), département et le type d'activité agricole dominant de la commune. 2600 micro-zones, dont 500 en montagne ont été définies. Le contexte local dans lequel s'insère chaque exploitation laitière a ainsi pu être assez finement précisé.

- les micro-bassins (de un à cinq par département) s'appuient, selon les zones, sur le zonage AOP, PRA ou sur des seuils de densité laitière. Ces 43 micro-bassins permettent de caractériser les dynamiques laitières et les enjeux de développement à une échelle pertinente pour les acteurs du développement.

La construction de ces deux échelles d'analyse et le travail à partir de données individuelles exhaustives constitue une avancée importante dans la caractérisation des systèmes de production laitiers de montagne. Les travaux réalisés jusqu'à présent s'appuyaient en effet sur les données du Réseau d'Information Comptable Agricole (RICA) et ne permettaient pas une caractérisation allant au-delà de six regroupements de PRA (Perrot et al 2008). 
nombre d'ateliers laitiers (- 34\% de 1999 à 2009), mais par un développement moindre des formes sociétaires. Les modalités de restructuration sont spatialement différenciées. La dynamique est particulièrement favorable dans le massif du Jura : les cessations sont limitées (-20\% de producteurs), la modernisation des exploitations et les formes sociétaires fréquentes. A l'autre extrême, la déprise est marquée $(-43 \%$ de producteurs) dans les Pyrénées, les zones peu denses du Massif Central et les Alpes hors zones AOP.

Une analyse des déterminants individuels et territoriaux des cessations laitières a été réalisée à l'aide d'un travail de modélisation économétrique (Dervillé 2012). Il apparaît ainsi qu'au côté de la taille des exploitations et de l'organisation en forme sociétaire, les économies de gamme (vente directe et services environnementaux) augmentent les chances de survie des exploitations. Le rôle du contexte territorial (micro-zones laitières) dans la recomposition du tissu laitier est également mis en évidence. Les stratégies de segmentation de l'aval, qu'elles soient génériques (profil produit des entreprises) ou spécifiques (AOP), apparaissent déterminantes de la dynamique laitière. Enfin, les risques de déprise sont mis en évidence dans les zones laitières peu denses : la décision de cessation d'un voisin constitue en effet une incitation à cesser l'activité, alors que sur les territoires plus denses elle est perçue comme une opportunité et favorise la poursuite de l'activité.

Ainsi, l'analyse des dynamiques laitières tend à montrer que les perspectives de poursuite de l'activité varient avec les ressources territorialement disponi-

Tableau 2. Caractéristiques des départements d'étude.

\begin{tabular}{|c|c|c|c|}
\hline & 1-Haute-Loire & 2-Cantal & 3-Doubs \\
\hline Nombre de producteurs & 2178 & 2310 & 1982 \\
\hline Quota moyen (L) & 177175 & 181131 & 192205 \\
\hline Spécialisation laitière & $\begin{array}{c}\text { récente } \\
\text { (après 1950) }\end{array}$ & $\begin{array}{l}\text { ancienne (nord) } \\
\text { récente (sud) }\end{array}$ & ancienne \\
\hline Technologie de production & $\begin{array}{l}\text { intensification } \\
\text { partielle }\end{array}$ & $\begin{array}{l}\text { intensification } \\
\text { partielle }\end{array}$ & herbager \\
\hline Poids des filières AOP & négligeable & $60 \%$ & $95 \%$ \\
\hline Profil des transformateurs & $85 \%$ nationaux & $65 \%$ nationaux & $75 \%$ locaux \\
\hline Technologie d'aval & industrielle & $\begin{array}{l}\text { partiellement } \\
\text { industrialisée }\end{array}$ & artisanale \\
\hline
\end{tabular}

bles ; externalités liées à la densité laitière et potentiel de valorisation AOP notamment. Quatre principales configurations productives peuvent ainsi être distinguées en montagne : zones à $\mathrm{AOP}$ fortes, zones à potentiel AOP, zones denses à destination de marchés génériques, zones génériques peu denses. Quels sont les enjeux soulevés par la suppression des quotas sur chacun de ces ensembles ? La libéralisation des marchés exerce en effet des pressions contrastées sur les différents régimes de concurrence associés à ces configurations. Les stratégies individuelles et collectives qui les sous-tendent et les condi-
Carte 4. Nombre d'AOP (fromages de vache) par commune (Données : INAO).

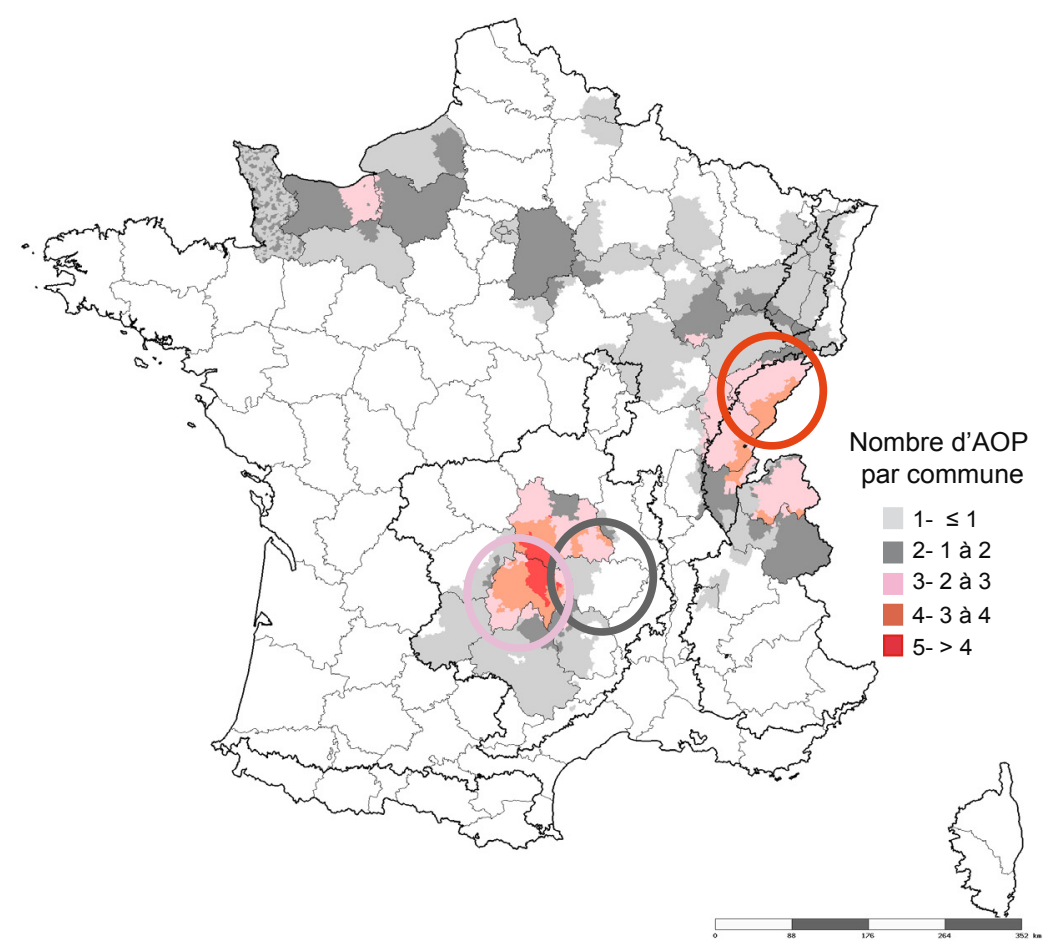

tions d'accès au marché des producteurs qui en découlent sont analysées, dans cette perspective, avant et après le tournant libéral de 2008, dans trois départements contrastés.

\section{3 / Un potentiel post-quotas d'ancrage territorial de la production variable selon les régimes de concurrence}

\section{1 / Diversité des régimes régio- naux de concurrence avant 2008}

Le Doubs représente les zones à AOP fortes caractérisées par un régime de concurrence fromager, le Cantal les zones à potentiel AOP caractérisées par un régime de concurrence hybride et la Haute-Loire les zones hors AOP sous régime de concurrence laitier (carte 4 et tableau 2). Le type de ressources mises en commun, leur modalité de gestion et les droits des agriculteurs sur ces ressources sont explicités pour chaque cas. Le système d'institutions spécifique à chaque régime de concurrence peut ainsi être défini.

a) Régime de concurrence laitier (castype: Haute-Loire)

Le développement de la spécialisation laitière altiligérienne date de la politique structurelle des années 1960. Les technologies intensives (révolution fourragère, adoption de races laitières spécialisées) ont alors été adoptées dans la limite des contraintes pédoclimatiques. La collecte et la transformation en pro- 
duits laitiers ont été confiées à des industries de statut privé ou coopératif. La capacité d'innovation des acteurs départementaux repose sur une capacité d'adoption de savoirs agronomiques et industriels génériques développés à l'échelle nationale : races à haut-potentiel, révolution fourragère et ration alimentaire pour la production laitière ; standardisation et économies d'échelle pour la transformation. La convention de qualité qui régie l'innovation est industrielle : le lait est apte à toute transformation et les produits laitiers répondent à des critères de qualité standard.

En 2006, les acteurs de la collecte et de la transformation de Haute-Loire sont majoritairement extra-départementaux ou nationaux. Les laits collectés ne sont pas nécessairement transformés dans le département. Un tiers du lait est d'ailleurs collecté par une coopérative de vente de lait de la région lyonnaise (URCVL) qui ne dispose pas d'outil de transformation. En outre, le département est dépourvu de filière AOP. Ainsi, la filière laitière du département est générique et peu territorialisée.

Le régime de concurrence est qualifié de laitier dans la mesure où les conditions d'accès des éleveurs aux marchés tiennent à l'organisation des échanges de lait standard. Les principales structures de gouvernance sont professionnelles (syndicat majoritaire, Commission Départementale d'Orientation Agricole -CDOA- et interprofession laitière régionale) et gèrent des ressources génériques (droits à produire, grille standard de paiement du lait à la qualité). La rémunération des éleveurs repose d'abord sur leur capacité individuelle à fournir un lait dont les caractéristiques physicochimiques et bactériologiques respectent les standards de qualité industrielle. Elle tient aussi à leur capacité collective à négocier, au sein des instances interprofessionnelles, les critères pris en compte et la valeur (pénalité ou prime de qualité) qui leur est attribuée dans la grille de paiement du lait à la qualité. La valeur minimale du lait standard est garantie par le rationnement de l'offre et les outils de protection aux frontières.

En l'absence d'AOP et compte-tenu de la faible territorialisation des entreprises d'aval, l'ancrage de la production repose sur la gestion départementalisée de la ressource générique « quota ».

b) Régime de concurrence fromager (cas-type : Doubs)

La spécialisation laitière du Doubs tient à une tradition d'élevage et de fabrication fromagère ancienne. En outre, les opérateurs du secteur ont fait le choix, de longue date, de développer la pro- duction en valorisant les ressources spécifiques locales : race locale, ressources herbagères, travail du lait cru, savoirfaire artisanaux. La répartition des tâches entre opérateurs de la filière est spécifique. Les éleveurs sont restés engagés dans l'aval. $75 \%$ du lait est transformé au sein de fruitières : petites coopératives fromagères dont les éleveurs sont propriétaires et continuent d'assurer la gestion. La maturation et la mise en marché des fromages sont réalisées par des maisons d'affinage qui ont, pour une large part, conservé un caractère régional $(45 \%$ de la mise en marché).

Le régime de concurrence est qualifié de fromager dans la mesure où la filière est structurée autour de l'appellation Comté, première appellation française par son tonnage. Le département compte deux autres appellations (Morbier et Mont d'Or), plus modestes en volume et dont les modalités de gestion sont bien articulées avec celles de l'AOP Comté (les conditions de production du lait notamment sont similaires). $95 \%$ du lait de montagne est ainsi valorisé dans des filières AOP. Les opérateurs économiques sont fédérés par un projet commun : «produire du Comté de qualité et renforcer la réputation du produit ». Les acteurs de la filière se sont dotés de structures de gouvernance spécifiques s'appuyant sur le cadre juridique protecteur reconnaissant les AOP et s'articulant avec le système institutionnel du régime de quotas.

Ces structures de gouvernance sont spécifiques et territorialisées. La première d'entre elle est le cahier des charges. Il définit les conditions de production et, par là même, les identités des opérateurs de marché. Le renforcement de ce cahier des charges au fil du temps a permis à la filière d'innover tout en préservant son identité. L'investissement d'opérateurs nationaux dans la filière dans les années 1990 a ainsi conduit à une extension du marché sans dégradation de sa réputation. La limitation du rayon de collecte à $25 \mathrm{~km}$ a notamment empêché la concentration de la production. Le Comité Interprofessionnel du Gruyère de Comté (CIGC) créé en 1963 a deux missions principales : garantir la qualité du produit aux consommateurs et assurer aux opérateurs la pérennité de leur activité économique. Etant autorisé à faire appliquer les décisions prises à l'unanimité du conseil d'administration à l'ensemble de ses membres, le CIGC a doté la filière de nombreux dispositifs de coordination complémentaires au cahier des charges. Plaques d'identification des fromages et dispositifs de contrôle permettent de garantir la traçabilité et la qualité des fromages. Le financement de programmes de recherche, la promotion et les activités de défense de l'appellation renforcent la capacité locale d'innovation et la réputation des fromages. La mise en place d'un système d'information, d'un plan de campagne et de règles d'évaluation de la valeur ajoutée créée aux différents stades de la filière permettent d'assurer le suivi des marchés, d'adapter l'offre à la demande et d'assurer une répartition équilibrée de la valeur ajoutée.

Ainsi, tous les opérateurs de la filière participent à la construction et à la gestion d'un système de ressources communes territorialisées (réputation et capacité d'innovation). Ils bénéficient d'un accès à des ressources spécifiques (compétences techniques et organisationnelles, usage du nom, prix stable et rémunérateur). Les conditions d'accès au marché des éleveurs tiennent à leur capacité individuelle à se conformer au cahier des charges, ainsi qu'à leur capacité collective à participer au pilotage des filières AOP (évolution du cahier des charges, orientation des programmes de recher-

Figure 1. Niveau de valorisation du lait dans les trois départements d'étude (Dervillé 2012, Données Agreste).

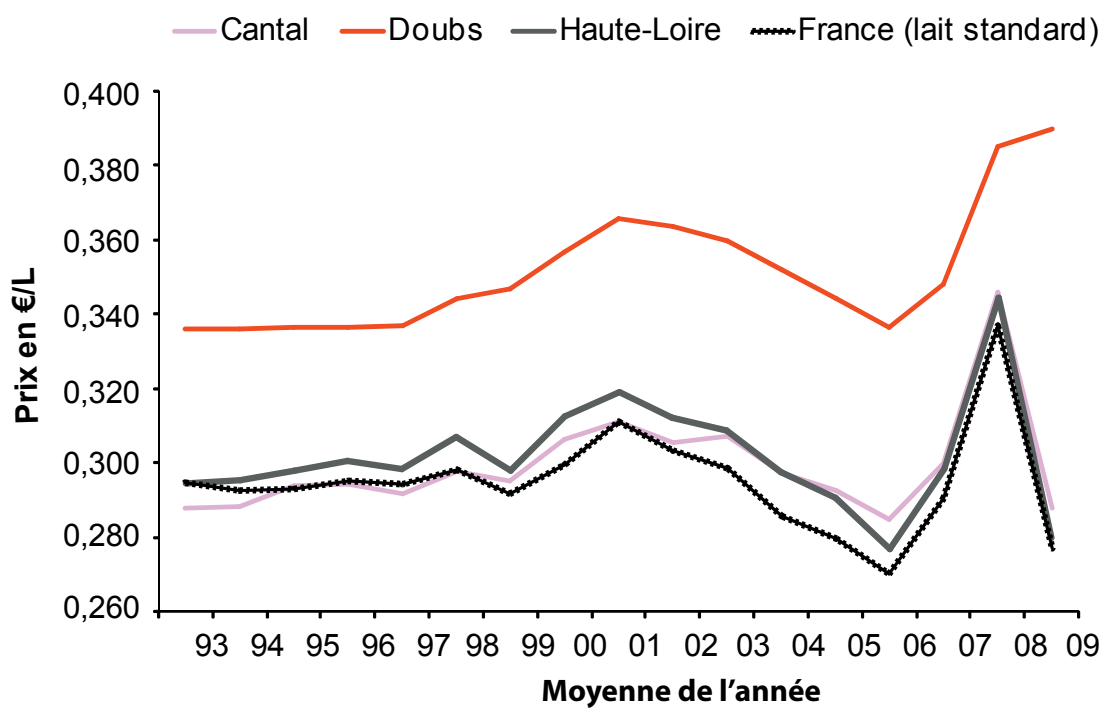


che) et à négocier une part de la valeur ajoutée créée. Ils ont bénéficié sur une longue période d'un prix du lait supérieur de $20 \%$ à la moyenne nationale (figure 1).

La principale échelle d'élaboration des modalités de contrôle de la concurrence est régionale, elle est liée à l'aire d'appellation. La territorialisation de la production est assurée par la différenciation des ressources productives et des marchés.

\section{c) Régime de concurrence hybride (cas-type : Cantal)}

Le Cantal a un profil contrasté au regard du développement de la production laitière. Le nord-ouest du département, zone herbagère d'altitude, est une zone historique de fabrication fromagère sous appellations (Cantal, Salers, Saintnectaire, Bleu d'Auvergne et Fourme d'Ambert). Néanmoins, dans les années 1960, le développement laitier a reposé sur l'abandon des races mixtes traditionnelles (Salers et Aubrac) et sur l'adoption de technologies intensives. Les agriculteurs se sont en outre largement désengagés de la transformation et de la commercialisation des produits. Cette évolution a été permise par le caractère peu contraignant des cahiers des charges des appellations. Elle a aussi été entrainée par un développement de la production excédant le potentiel de valorisation AOP. L'est et surtout le sud du département, historiquement peu laitiers, se sont en effet spécialisés dans la production laitière. Des coopératives ont été créées et ont investi dans le développement de filières laitières génériques (fromage, beurre, lait). Au fil des opérations de restructuration, filières génériques et AOP se sont rapprochées, jus- qu'à être gérées sur les mêmes sites de fabrication.

Dans le Cantal, des ressources spécifiques existent (savoir-faire herbagers et fromagers, structures de gouvernance spécifiques : Comité Interprofessionnel des Fromages (CIF), Pôle fromager AOP Massif Central), mais les conventions de production génériques et industrielles se sont imposées. La capacité d'innovation amont repose sur les structures professionnelles : syndicat, Chambre d'agriculture, CDOA, laboratoire interprofessionnel. Il en résulte une capacité d'innovation plus sectorielle que territoriale. Jusqu'en 2009, les éleveurs ne connaissaient pas le devenir de leur lait et ne bénéficiaient pas de prime AOP. Comme dans le régime laitier, le prix du lait est fixé dans le cadre des accords interprofessionnels nationaux.

Les stratégies d'innovation de l'aval sont pour la plupart internes aux entreprises. Hors fromages fermiers, la valeur ajoutée éventuellement créée en lien avec la réputation des produits est appropriée par des transformateurs de plus en plus concentrés. En 2006, les deux premiers opérateurs, extérieurs au département, assuraient $70 \%$ de la transformation; en 2011 ce pourcentage s'élevait à $90 \%$.

Ainsi, les conditions d'accès des éleveurs aux marchés reposent plus sur des dispositifs de coordination sectoriels nationaux que sur des accords territorialisés de coopération entre opérateurs des filières de fromages sous appellation. Le système de ressources communes à l'origine de la stabilité des marchés est essentiellement sectoriel et générique.

Au final, sous régime de quotas, les ressources activées par les opérateurs des régimes laitier et hybride sont génériques. L'ancrage de la production est assuré par la gestion départementalisée des quotas. La comparaison des castype du Cantal et du Doubs montre que les AOP ne contribuent véritablement au renforcement de la compétitivité horscoût de l'ensemble des opérateurs de la filière, et donc à l'ancrage de la production, que lorsque les opérateurs se sont dotés d'un système de ressources spécifiques territorialisées qui leur permet d'innover et de créer de la valeur. La participation des éleveurs à la gestion de ce système de ressources communes est une condition d'accès aux ressources ainsi constituées (compétences spécifiques et prime de qualité).

\section{2 / Des capacités d'adaptation à l'après-quotas contrastées}

La hausse des quotas laitiers jusqu'à leur suppression correspond à une modification $\mathrm{du}$ fonctionnement des marchés laitiers à plusieurs échelles : européenne (affaiblissement de la protection du marché européen et diffusion de l'instabilité des prix), États membres et départements (renforcement de la concurrence entre producteurs et entre territoires) et opérateurs (fin d'un accès au marché et d'approvisionnements garantis). Ainsi le désengagement de l'État et de l'Europe de la régulation des marchés laitiers déstabilise les interactions entre acteurs.

L'analyse des réactions et des stratégies d'adaptation mises en œuvre dans chacun des départements d'étude sur la période 2008-2011 met en évidence le rôle du système initial de ressources dans cellesci (tableau 3).

Tableau 3. Ressources communes et stratégies d'innovation selon le régime de concurrence.

\begin{tabular}{|c|c|c|c|}
\hline $\begin{array}{l}\text { Régime de } \\
\text { concurrence }\end{array}$ & $\begin{array}{c}\text { Laitier } \\
\text { (Cas-type : Haute-Loire) }\end{array}$ & $\begin{array}{c}\text { Hybride } \\
\text { (Cas-type: Cantal) }\end{array}$ & $\begin{array}{c}\text { Fromager } \\
\text { (Cas-type : Doubs) }\end{array}$ \\
\hline \multirow{2}{*}{$\begin{array}{l}\text { Ressources } \\
\text { communes }\end{array}$} & Savoir- & $\begin{array}{l}\text { re génériques } \\
\text { Ressources spécifiques } \\
\text { partiellement activées }\end{array}$ & $\begin{array}{l}\text { Savoir-faire et dispositifs de } \\
\text { coordination spécifiques }\end{array}$ \\
\hline & \multicolumn{2}{|c|}{$\begin{array}{l}\text { Capacités d'innovation sectorielle } \\
\text { Rente liée aux quotas }\end{array}$} & $\begin{array}{l}\text { Capacité d'innovation } \\
\text { territorialisée et distribuée } \\
\text { Valeur ajoutée des fromages }\end{array}$ \\
\hline $\begin{array}{l}\text { Impact de la } \\
\text { libéralisation }\end{array}$ & \multicolumn{2}{|c|}{ Marqué } & Atténué \\
\hline $\begin{array}{l}\text { Réponses } \\
\text { locales I } \\
\text { Innovations }\end{array}$ & $\begin{array}{l}\text { Logique industrielle } \\
\text { et corporatiste } \\
\text { - Demande d'intervention } \\
\text { de l'Etat } \\
\text { - Concentration des } \\
\text { entreprises } \\
\text { - Création de marques }\end{array}$ & $\begin{array}{l}\quad \text { Tournant Qualitatif } \\
\quad \text { - Révision du cahier des } \\
\text { charges } \\
\text { - Certification des opérateurs } \\
\text { - Négociation d'une prime AOP } \\
\text { pour les producteurs } \\
\text { - Renforcement des structures } \\
\text { de gouvernance AOP }\end{array}$ & $\begin{array}{l}\text { Logique qualitative renforcée } \\
\text { Renforcement des dispositifs } \\
\text { de coordination: } \\
\text { - Droits à produire des } \\
\text { fromages ramenés à la surface } \\
\text { - Capacité territoriale } \\
\text { d'investissement } \\
\text { - Diversification... }\end{array}$ \\
\hline
\end{tabular}


Le régime fromager a été peu affecté par la libéralisation des marchés laitiers. Le système de ressources communes a préservé la rente de qualité malgré les instabilités du marché mondial, comme en témoigne la croissance du prix du lait entre 2007 et 2009 (figure 1). La stratégie collective d'adaptation au changement de contexte politique et économique comporte trois volets principaux :

la préservation de l'identité de la filière lors de la révision du cahier des charges en 2007 (système de production extensif, limitation des possibilités de croissance des fruitières),

- le renforcement des structures de gouvernance de l'appellation, pour qu'elles survivent à la suppression des quotas (transformation du plan de campagne avec adossement du droit à produire du fromage non plus aux quotas mais à la surface),

- l'accroissement de la valeur ajoutée (investissement dans la recherche sur la qualité des produits, développement de marchés à l'export et création d'un GIE lait comtois pour gérer les laits à Comté non valorisables sous appellation).

La longue expérience de l'action collective a ainsi permis aux acteurs de ce régime de se doter de nouveaux dispositifs de coordination pour adapter leur capacité de contrôle de la concurrence au changement d'ordre institutionnel.

$\mathrm{Au}$ contraire, le régime laitier a été fortement déstabilisé par la libéralisation des marchés. Le profil des opérateurs de la collecte et de la transformation du lait sur le département expose le système de production haut-ligérien à la diffusion des instabilités mondiales (flexibilité pour le groupe Sodiaal et faillite de l'URCVL). Les producteurs se sont ainsi retrouvés confrontés à une baisse du prix du lait, voire à une incertitude sur le maintien de la collecte, dans un contexte de coût des intrants élevé et, pour certains d'entre eux, dans une situation d'endettement liée à des investissements récents. Cependant, la stratégie d'adaptation reste marquée par une logique sectorielle et corporatiste. Les producteurs se sont mobilisés pour que l'État intervienne et favorise la recherche de solutions pour assurer le maintien de la collecte. Elle a été maintenue mais cette intervention renforce le pouvoir décisionnel de l'acteur public et des entreprises dans le contrôle de l'évolution structurelle du secteur. Les incitations à la concentration des structures de production sont fortes. Ayant pris conscience du risque de perte de capacité collective de contrôle de leur devenir, les représentants syndicaux s'organisent pour activer de nouvelles ressources. Ils tentent de se réapproprier la dénomination Montagne par la création d'une marque collective, détenue par les éleveurs, dans un objectif d'ancrage des volumes et potentiellement de création de valeur ajoutée.

Dans le Cantal (régime hybride), la perte de compétitivité des systèmes génériques de montagne a conduit les opérateurs à revoir leur modèle de développement. Des leaders locaux sont parvenus à mobiliser les éleveurs et les élus locaux autour d'un projet collectif de réappropriation et de relance des filières AOP. Des ressources spécifiques jusque là peu mobilisées (savoir-faire herbager, structures de gouvernance des AOP) ont été activées. Le renforcement de la politique de la qualité et la réforme des modalités de certification des indications géographiques ont encouragé cette dynamique. De nouvelles règles ont été élaborées. Le renforcement du cahier des charges (définition de conditions de production du lait visant à renforcer le lien au terroir et allongement de la durée d'affinage) et l'obligation de certification de tous les opérateurs de la filière ont permis aux producteurs de lait AOP d'accéder pour la première fois en 2009 à une prime de qualité AOP. Cette initiative n'est pas encore pérenne car elle repose sur une Cotisation Volontaire Obligatoire (CVO) non-conforme au regard du droit européen. Elle a néanmoins permis de financer des actions de promotion et un soutien aux producteurs. Cette dynamique collective a incité les institutions sectorielles (Chambre d'agriculture, Contrôle laitier, laboratoire interprofessionnel...) à se rapprocher des institutions fromagères territorialisées (CIF, Pôle fromager, INRA d'Aurillac) pour accompagner les exploitations laitières dans la mise en conformité de leur système de production avec les nouvelles exigences du cahier des charges. Cette évolution structurelle s'accompagne de la constitution d'une capacité d'innovation régionale qui peut permettre l'émergence de solutions territorialisées face à la perte de compétitivité du système régional de production.

Ainsi, dans le régime fromager, la différenciation des systèmes productifs et la capacité d'innovation territorialisée atténuent les effets de la libéralisation. $\mathrm{Au}$ contraire, les régimes de concurrence génériques et hybrides, dans lesquels les quotas jouaient un rôle essentiel à la coordination des acteurs, sont fortement déstabilisés. La possibilité d'activer des ressources spécifiques favorise la transformation du régime de concurrence hybride. De la comparaison des trois régimes régionaux de concurrence, il ressort un enjeu de structuration de l'action collective pour créer de la valeur et stabiliser les marchés. Le succès des filières AOP du Doubs suggère que c'est la capacité collective à générer une certaine rareté (réputation et gestion de l'offre), via l'élaboration d'un système d'institutions, qui permet aux éleveurs de participer à la gestion d'une stratégie de différenciation et de bénéficier d'une part de valeur ajoutée. Plusieurs innovations institutionnelles pertinentes ont été mises en évidence:

- La construction d'une identité et d'une réputation, valorisant les spécificités des pratiques locales, permet une segmentation des marchés et de l'espace de concurrence,

- L'élaboration d'un cahier des charges et la mise en place d'une procédure de traçabilité offre une garantie aux consommateurs et contribue au renforcement de la réputation par la stabilisation d'une qualité. En répartissant les responsabilités, ces institutions permettent d'assurer un pouvoir de négociation à chacun des opérateurs de la filière,

- Les instruments de suivi des marchés et de maîtrise des volumes permettent de stabiliser les prix et d'assurer une répartition équilibrée de la valeur ajoutée,

- La recherche, l'innovation et la promotion renforcent l'identité d'un produit en l'adaptant à l'évolution de la réglementation publique et des modes de consommation.

Le tournant qualitatif mis en œuvre dans le Cantal est particulièrement instructif à ce titre. Il met clairement en évidence que l'élaboration de règles collectives est à l'origine de nouveaux droits individuels : une prime de marché de l'ordre de $20 € / t$ et un accès à une capacité d'innovation spécifique, dans le cas présent. La compétitivité des exploitations laitières des filières AOP s'en trouve potentiellement renforcée. Ce cas-type met également en évidence que les capacités collectives d'innovation dépendent à la fois de la capacité des opérateurs à fédérer leurs intérêts et de l'évolution du cadre institutionnel macro-économique.

De la caractérisation des trois principaux régimes de concurrence, il ressort qu'en fonction du système initial de ressources, les capacités d'adaptation à l'après-quotas des filières et des territoires sont contrastées. La combinaison de cette étude de cas à l'analyse statistique des déterminants de la restructuration laitière permet de cartographier les enjeux d'adaptation à l'après-quotas sur l'ensemble de l'espace montagnard.

\section{4 / Cartographie des régimes régionaux de concurrence et des enjeux d'adaptation à l'après-quotas en montagne}

Le regroupement, à l'intérieur des départements, de communes laitières 
Carte 5. Typologie des micro-bassins laitiers de montagne (Dervillé 2012).

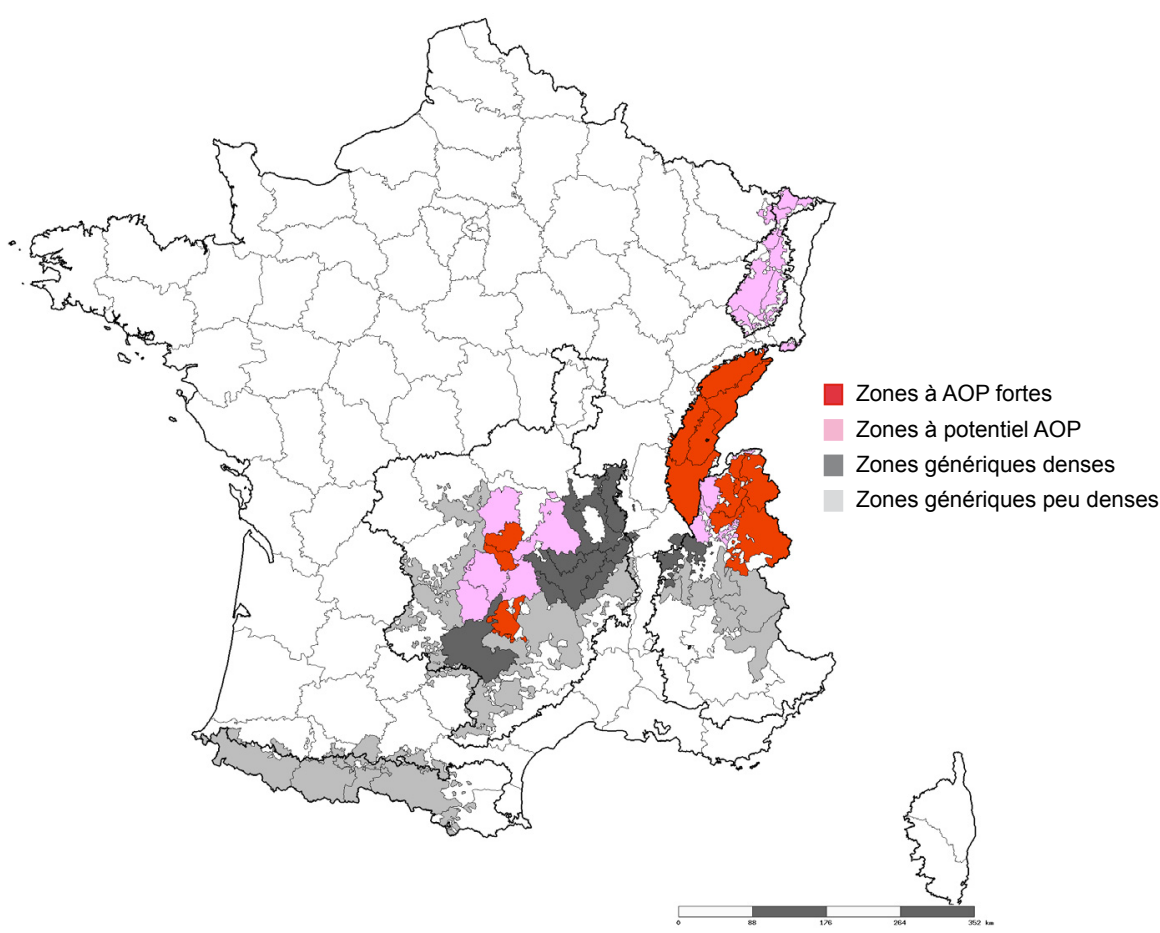

contiguës homogènes au regard des contraintes de milieu (sur la base du zonage ICHN et petites régions agricoles PRA), de la densité laitière (seuil à $40000 \mathrm{~L} / \mathrm{km}^{2}$ ) et du profil AOP (absence, présence, débouché majoritaire) a conduit à la construction de 43 micro-bassins de montagne (3-4 zones par département) (encadré 2). Ces derniers peuvent être regroupés en quatre grands ensembles correspondant aux quatre configurations productives majeures de la production laitière de montagne (carte 5).

La suppression de la ressource générique « quota ", dont les modalités de gestion assuraient l'ancrage des volumes à l'échelle des départements, incite les acteurs sectoriels et territoriaux à coopérer dans la construction et la consolidation de ressources alternatives permettant de séparer les espaces de concurrence. Compte-tenu des contraintes et des surcoûts qui rendent difficile la concurrence sur les coûts de production, il s'agit pour les opérateurs économiques de coopérer dans la constitution et la gestion de ressources spécifiques pouvant servir de base à la différenciation des modèles productifs et des marchés, et renforcer ainsi leur compétitivité hors-coût.

En créant de la valeur (marques d'entreprises) et/ou en rendant la production impossible à délocaliser (AOP, IGP, dénomination montagne, circuits-courts), les stratégies de segmentation des marchés peuvent permettre de renforcer la compétitivité des filières de montagne et d'assurer leur viabilité sur le long terme. La création de valeur en aval ne constitue pas cependant une garantie pour les éle-
Pour les éleveurs concernés $(27 \%$ des producteurs de montagne en 2009), l'enjeu collectif repose sur le renforcement de ces dispositifs.

Dans les zones à potentiel AOP (régime hybride), l'enjeu collectif d'adaptation repose sur l'activation de ressources spécifiques permettant de renforcer la réputation des fromages AOP et d'améliorer la gestion de l'offre. Pour pouvoir exercer un contrôle sur le renforcement de ces démarches de qualité et en bénéficier, les producteurs de ces zones $(25 \%$ des producteurs de montagne), devraient élargir leurs compétences collectives à la compréhension du fonctionnement des filières et des marchés AOP. Ces compétences nouvelles leur permettraient de prendre activement part au fonctionnement des structures de gouvernance existantes (Organisme de défense et de gestion des AOP). La poursuite de travaux de recherche sur les liens entre pratiques, qualité du lait et qualité des produits, permettrait de renforcer la capacité d'innovation et la réputation des filières AOP et d'objectiver le rôle des producteurs dans ces deux processus; leur ouvrant ainsi un accès durable à une rente de qualité territoriale.

veurs. Les entreprises n'entendent nécessairement partager leur profit avec les éleveurs et payer le lait plus cher (Dervillé 2012, Chatellier et al 2013). En outre, la substitution de contrats aux quotas, renforce la capacité des entreprises à choisir leurs partenaires (et donc à exclure des producteurs). L'analyse des régimes de concurrence fromager et hybride après 2008 montre que la possibilité, pour les éleveurs, de négocier une part de la valeur ajoutée et d'exercer un contrôle collectif sur les conditions d'accès au métier, résulte d'une contribution objectivée à la compétitivité des filières. Cela peut passer $i$ ) par un renforcement de leur responsabilité collective dans la maîtrise de la qualité des produits via la certification notamment ou, ii) par le développement d'une capacité collective à gérer une offre de lait (qualité et volume) à l'échelle de fonctionnement des bassins de production pour offrir des services pertinents à l'aval (adaptés à la demande). Il peut s'agir de développer des outils de gestion des volumes par campagne et d'optimiser les circuits de collecte (basculement de la production de cantons contigus dans la production de lait bio ou AOP par exemple pour réduire les coûts de collecte).

Selon les configurations productives, les ressources sur lesquelles les producteurs peuvent s'appuyer pour participer au pilotage des filières sont contrastées.

Dans les zones à AOP fortes (régime fromager), la réputation des produits et les dispositifs de coordination spécifiques atténuent l'impact de la libéralisation.
Dans des zones génériques denses (régime laitier), les dispositifs de coordination pouvant permettre une différenciation des formes et des espaces de concurrence après la suppression des quotas restent à construire. Pour $30 \%$ des producteurs de montagne situés dans ces zones, le réinvestissement de l'aval est également primordial. Dans ce cas cependant, la participation au pilotage des filières passe par la constitution d'organisations de producteurs. Elles peuvent être le support de la négociation du prix du lait mais aussi de la co-construction de stratégies de segmentation, en partenariat avec les entreprises laitières (dénomination montagne, lait à l'herbe ou « tout foin », marques régionales).

Dans les zones génériques peu denses, les enjeux se rapprochent de ceux des zones génériques denses lorsqu'un site de transformation existe à proximité (12\% des producteurs). Dans le cas contraire (6\% des producteurs), les possibilités de réponse collective sont compromises. Le développement de la fabrication fermière ou à petite échelle apparaît comme le principal levier de préservation de la production.

Le cas du régime fromager du Comté, ayant acquis une forte autonomie, n'est pas généralisable à l'ensemble des zones de montagne où interviennent en général des acteurs locaux et nationaux, coopératifs et privés, présents sur différents marchés. La résistance des systèmes laitiers de montagne repose donc sans doute 
sur la convergence de plusieurs stratégies. Ainsi, les producteurs ont-ils collectivement intérêt à se saisir de la possibilité de s'organiser sur une base territoriale large. Les neuf bassins définis par l'interprofession laitière, comme échelle de gestion des quotas jusqu'à leur suppression, pourraient a minima servir de base à la coordination entre les collectifs d'éleveurs et les industriels. Chaque bassin de production pourrait ensuite s'organiser en sections pour gérer un système de ressources communes particulier: AOP, agriculture biologique, dénomination montagne, production fermière, marques régionales, circuits-courts. La gestion coordonnée de ces sections est souhaitable car c'est l'articulation entre marchés qui permet de gérer les volumes. Si les organisations de producteurs parviennent à se placer à l'articulation entre secteurs et territoires, il leur sera vraisemblablement plus facile de retenir de la valeur en amont de la filière.

Les organisations de producteurs peuvent aussi créer de la valeur en élargissant leur offre à la production de services écologiques à destination d'acteurs publics ou privés. Plus largement, la capacité collective des producteurs de montagne à négocier une part conséquente de l'enveloppe budgétaire de la nouvelle PAC, que cela soit pour prendre en charge le handicap ou rémunérer les services environnementaux, constitue également un enjeu.

Enfin, un troisième domaine de différenciation des modèles productifs concerne la diversification des activités au sein des exploitations agricoles de montagne. Elle peut permettre d'asseoir leur viabilité sur des bases différentes des exploitations de plaine : tourisme, énergie, forêt.

\section{Conclusion}

Le modèle conceptuel du fonctionnement des marchés ainsi que la combinaison d'une analyse statistique et d'une analyse des stratégies d'acteurs pour l'informer ont permis de proposer une vision renouvelée et enrichie des enjeux de l'adaptation à l'après-quotas en montagne.

Compte-tenu des contraintes pédoclimatiques sur la possibilité de gain de productivité, la différenciation est une stratégie essentielle d'adaptation à la libéralisation des marchés.

De l'analyse en termes de régimes de concurrence, il ressort que la différenciation des systèmes productifs et des marchés repose sur l'élaboration de règles de choix collectifs encadrant les comportements individuels, et permettant l'activation et la gestion durable de ressources matérielles et immatérielles spécifiques. L'implication des éleveurs dans ce processus de spécification et leur participation à l'élaboration des règles de choix collectifs sont essentielles car elles conditionnent leurs droits de gestion et d'accès aux ressources créées. En d'autres termes, c'est en participant à la gouvernance du système de ressources à l'origine de la différenciation du régime de concurrence, que les producteurs peuvent exercer un contrôle collectif sur leurs conditions d'accès au marché et donc sur l'évolution de leur métier. La capacité collective à gérer une offre de lait spécifique (quantité et qualité) est le principal système de ressources que les producteurs peuvent activer pour participer, au côté des entreprises, à l'élaboration de stratégies de valorisation du lait. C'est à cette condition qu'ils pourront exercer une responsabilité dans le pilotage des filières et accéder à une rente de qualité territoriale qui permettra de les protéger de la concurrence des systèmes de plaine.

Si tel n'est pas le cas, suite à la disparition de la ressource générique quota, la concurrence sur les coûts sera renforcée, le rôle des entreprises dans la sélection des producteurs d'avenir accentué, et la restructuration subie.

L'adoption du Paquet Lait, en reconnaissant les interprofessions, en favorisant la contractualisation et la constitution d'organisations de producteurs fortes et en autorisant la maîtrise de la croissance des volumes dans les filières AOP, constitue une incitation à la structuration de l'action collective. La réflexion du Ministère de l'Agriculture sur les groupements d'intérêt économiques et écologiques élargit ce processus à la production de services écologiques et territoriaux. Toutefois, le développement de telles démarches collectives de construction d'une offre de produits laitiers et de services demande du temps et des investissements conséquents, matériels (programmes de recherche, promotion, réseaux logistiques) et immatériels (valeurs, savoir-faire, dispositifs de coordination). Or, la libéralisation du marché laitier suisse (Kroll et al 2010) a montré la difficulté, pour les opérateurs économiques, de se substituer à l'État et de coopérer dans l'élaboration de dispositifs de stabilisation des marchés. Comme en France, les difficultés apparaissent d'autant plus grandes que les opérateurs ne bénéficient pas de systèmes de ressources communes alternatifs aux quotas. En Suisse, à la suite de la suppression des quotas en 2009, deux interprofessions ont en effet été mises en place. Alors que l'interprofession des fromages AOP et assimilés parvient à réguler les marchés spécifiques (les prix du lait restant rémunérateurs), l'interprofession du lait d'industrie peine à établir un rapport de force équilibré entre les opérateurs de la filière. Les producteurs ne parviennent pas à négocier des prix rémunérateurs. Malgré la possibilité de s'organiser en organisations de producteurs pour commercialiser collectivement leur lait, les producteurs sont restés trop divisés pour pouvoir peser face à un aval concentré. D'une part, les comportements opportunistes ont réduit la capacité collective à gérer les volumes. D'autre part, les organisations de producteurs sont souvent restées sectorielles (liées à un industriel) et non territoriales, ce qui limite leur pouvoir de négociation face à l'aval. Ainsi, le cas suisse et les capacités contrastées d'adaptation des montagnes françaises, soulignent la difficulté pour les professionnels à investir dans la différenciation des systèmes productifs par l'activation de ressources spécifiques. Les capacités d'innovation territoriales se développent sur le temps long alors que l'échéance de 2015 est proche.

Pour développer rapidement une capacité collective à gérer une ou plusieurs offres spécifiques de lait, les producteurs et leurs représentants ont besoin d'être accompagnés. La mobilisation des pouvoirs publics à différentes échelles (européenne, nationale et régionale) est requise. De l'analyse des forces et des faiblesses des montagnes laitières européennes, il ressort une communauté d'intérêts dépassant la diversité des contraintes naturelles et des environnements économiques ainsi que la diversité des filières et des dynamiques de développement territorial, et qui est favorable à l'émergence de stratégies communes (Institut de l'Elevage 2009b, Perrot et al 2009). Il serait souhaitable que la possibilité pour des acteurs collectifs de gérer des marchés de qualité spécifique soit confortée par les politiques européennes de la concurrence et de la qualité. L'élargissement des stratégies de segmentation des marchés est également souhaitable. La reconnaissance d'une dénomination montagne à l'échelle européenne pourrait constituer un levier si elle s'appuie sur un cahier des charges exigeant correspondant aux attentes des consommateurs et aux possibilités d'adaptation des producteurs. La promotion et l'institutionnalisation des démarches de circuits courts constituent également une opportunité. Le cas des filières italiennes a montré que ce type de segmentation pouvait constituer, dans certaines zones touristiques ou densément peuplées, un facteur d'ancrage de la production. Plus largement, la promotion de la qualité spécifique auprès des consommateurs soutiendrait ces démarches. Lorsque les cahiers des charges contiennent des incitations à la mise en œuvre de pratiques extensives, l'attribution de soutiens publics, sur la 
base des zonages liés à la segmentation qualitative des marchés, pourrait également être envisagée (Dervillé et al 2012). Surtout, il serait pertinent que l'acteur public soutienne le développement de capacités d'innovation territoriales. Le principe de subsidiarité, en permettant de s'appuyer sur les politiques nationales et régionales, peut permettre de proposer une approche intégrée appropriée à la spécificité des différents massifs et des différents micro-bassins de montagne. Les pratiques de nos voisins européens en la matière peuvent constituer une source d'inspiration : soutiens régionaux à l'investissement de certaines régions italiennes et prise en compte de la diversité des handicaps de la montagne à l'instar des deux "Länder » du Sud de l'Allemagne, notamment. La for- mation des producteurs et du personnel des PME laitières et des organismes d'appui technique, à l'activation et à la gestion durable des ressources communes soutenant la différenciation des marchés, serait par ailleurs bénéfique. Si l'acteur public ne peut se substituer à l'organisation collective, il peut être proactif en soutenant, finançant et accompagnant l'émergence $i$ ) de démarches collectives de segmentation des marchés (étude de marché, expérimentation, promotion) et ii) des projets de restructuration (aide à l'investissement, remembrement, optimisation des circuits de collecte). La combinaison d'économies de gamme et d'économies d'échelle peut renforcer la compétitivité des systèmes laitiers de montagne et assurer leur maintien dans la durée.

\section{Remerciements}

Cet article s'appuie sur une thèse CIFRE réalisée à l'US ODR de l'INRA de Toulouse et cofinancée par la CNIEL. Les auteurs remercient Gilles Bazin (Professeur responsable) ainsi que les membres du comité de thèse, le département Economie et Qualité du CNIEL et l'équipe de l'ODR qui ont fortement contribué à l'aboutissement de ce projet. Les auteurs remercient également les relecteurs anonymes et les responsables de la revue pour leurs remarques constructives qui ont permis d'améliorer la qualité de l'article.

\section{Références}

Allaire G., 2002. L'économie de la qualité en ses secteurs, ses territoires et ses mythes. Geogr. Econ. Soc., 4, 155-180.

Allaire G., 2010. Applying economic sociology to understand the meaning of "Quality" in food markets. Agricult. Econ., 41, 167-190.

Boyer R., 1995. Secteurs, régions et modes de régulation. In : La grande transformation de l'agriculture. Boyer R., Allaire G. (Ed). Economica, Paris, France, 227-240.

Boyer R., 2003. Les institutions dans la théorie de la régulation. Cah. Econ. Pol., 44, 79-101.

Chatellier V., Delattre F., 2003. La production laitière dans les Alpes françaises: une dynamique particulière pour les Alpes du Nord. INRA Prod. Anim., 16, 61-76.

Chatellier V., Delattre F., 2006. Le prix du lait en Savoie et les filières fromagères AOC : principales évolutions passées et perspectives. Rapport Final GIS Alpes du Nord, 67p.

Chatellier V., Pflimlin A., 2007. Les systèmes laitiers des régions européennes de l'espace atlantique. In: European Workshop of the Green dairy project, Nantes, France, 23p.

Chatellier V., Guyomard H., 2009. Le Bilan de santé de la PAC et son application en France, simulations et réflexions suite aux décisions du 23 février 2009 du Ministre français de l'agriculture et de la pêche. In : Séminaire "José Rey" du Ministère de l'Agriculture et de la Pêche, INRA, Paris, France.

Chatellier V., Lelyon B., Perrot C., You G., 2013. Le secteur laitier français à la croisée des chemins. In : Numéro spécial, La vache et le lait. Faverdin P., Leroux C. (Ed). INRA Prod. Anim., 26, 77-100.

CNASEA, 2008. Evaluation ex post du Plan de Développement Rural National. Aides aux zones défavorisées. Rapport d'évaluation. Centre National pour l'Aménagement des Structures des Exploitations Agricoles. Marché CNASEA n²1-07, 23p.

Dervillé M., 2012. Territorialisation du secteur laitier et régimes de concurrence : le cas des montagnes françaises et de leur adaptation à l'après-quota. Thèse de Doctorat, AgroParisTech, Paris, France, 560p.

Dervillé M., Vandenbroucke P., Bazin G., 2012. Suppression des quotas et nouvelles formes de régulation de l'économie laitière : les conditions patrimoniales du maintien de la production laitière en montagne. Rev. Reg., 12, 21 p.

Fligstein N., 1996. Markets as politics: a political cultural approach to market institutions. Am. Soc. Rev., 61, 656-673.

Hess C., Ostrom E., 2003. Ideas, Artifacts, and Facilities: Information as a Common-Pool Resource. Law Contemp. Probl., 66, 111-146.

Institut de 1'Elevage, 2009a. France laitière 2015 : Vers une accentuation des contrastes régionaux. Le dossier Economie de l'Elevage, 391, 72p.

Institut de 1'Elevage, 2009b. Le lait dans les montagnes européennes, un symbole menacé. Le dossier Economie de l'Elevage, 390, 76p.

Kroll J.C., Trouvé A., Deruaz M., 2010. Quelle perspective de régulation après la sortie des quotas laitiers ? Faut-t-il encore une politique laitière? Rapport du CESEARn UMR 1041 INRA - Agrosup Dijon, France, 42p.

Lascoumes P., Le Gales P., 2004. L'action publique saisie par les instruments. In : Gouverner par les instruments, Lascoumes P., Le Gales P. (Ed). Presses de la fondation nationale des sciences politiques, 11-44.

Lelyon B., Chatellier V., Daniel K., 2012. Fin des quotas laitiers, contractualisation et stratégies productives: enseignements d'une modélisation bioéconomique. INRA Prod. Anim., 25, 67-76.

Ostrom E., 2000. Private and common property regimes. In: Encyclopedia of Law and Economics. Bouckaert B., De Geest G. (Ed). Edward Elgar, Cheltenham, England, 332-379.

Ostrom E., Schlager E., 1992. Property rights Regime and Natural resources: a conceptual analysis. Land Econ., 68, 249-262.
Paradis S., Denux J.P., Tocquer C., Sarrazin F., 2010. Avenir des zones à faible densité, Rapport FESIA, 2p.

Pecqueur B., 2001. Qualité et développement territorial: l'hypothèse du panier de biens et de services territorialisés. Econ. Rurale, 261, 37-49.

Perrat J., Zimmerman J.B., 2003. Stratégies des firmes et dynamiques territoriales. In : Entreprises et territoires. Dupuy C., Burmeister A. (Ed). La documentation française, Paris, France, 15-32.

Perrier-Cornet P., Sylvander B., 2000. Firmes, coordinations et territorialité. Une lecture économique de la diversité des filières d'appellation d'origine. Écon. Rurale, 258, 79-89.

Perrot C., Reuillon J.L., Capitain M., Cassez M., 2008. Forces et faiblesses des exploitations laitières françaises de montagne dans la perspective d'un desserrement de la contrainte des quotas laitiers. Renc. Rech. Rum., 15, 243-246.

Perrot C., Dervillé M., Monniot C., Richard M., 2009. Le lait dans les montagnes européennes. Un symbole menacé. Renc. Rech. Rum., 16, 215-218.

Ricard D., Rieutort L., 1995. Filières agro-alimentaires et moyennes montagnes françaises. Rev. Geogr. Alpine., 83, 101-124.

Touzard J.M., 1995. Régulation sectorielle, dynamique régionale et transformation d'un système productif localisé: exemple de la viticulture languedocienne. In : La grande transformation de l'agriculture. Allaire G., Boyer R. (Eds). INRA-Economica, Paris, France, 293-322.

Vandecandelaere E., Arfini F., Belletti G., Marescotti A., 2009. Linking people place and products. A guide for promotion quality linked to geographical origin and sustainable geographical indications. Rapport FAO, Rome, Italy, 218 p.

White H.C., Godart F.C., Corona V.P., 2008. Produire en contexte d'incertitude: la construction des identités et des liens sociaux dans les marchés. Sci. Soc., 73, 16-39. 
Annexe 1

Les bases de données mobilisées

\begin{tabular}{|c|c|c|c|}
\hline Fournisseurs & $\begin{array}{l}\text { Types de } \\
\text { données }\end{array}$ & Tables & Périodes \\
\hline \multirow{3}{*}{ FranceAgriMer } & \multirow{3}{*}{$\begin{array}{l}\text { Base } \\
\text { administrative de } \\
\text { gestion des } \\
\text { références laitières } \\
\text { (FAM) }\end{array}$} & $\begin{array}{l}\text { Identification des détenteurs de quotas } \\
\text { laitiers (exploitations) ( } 345000 \text { lignes) }\end{array}$ & \multirow{2}{*}{$\begin{array}{l}\text { Campagnes } 1999 / 2000, \\
2001 / 2002 \text { et } 2004 / 2005 \text { à } \\
2009 / 2010\end{array}$} \\
\hline & & $\begin{array}{l}\text { Notification des références (droits à } \\
\text { produire) par campagne (100 } 000 \text { lignes } \\
\text { en moyenne par campagne) }\end{array}$ & \\
\hline & & $\begin{array}{l}\text { Transfert foncier (achat/ vente de terre } \\
\text { avec quota) ( } 15000 \text { lignes par campagne) }\end{array}$ & $\begin{array}{l}\text { Campagnes 2004/ } 2005 \text { à } \\
\text { 2009/2010 }\end{array}$ \\
\hline \multirow[t]{2}{*}{$\begin{array}{l}\text { Service Central } \\
\text { des Enquêtes et } \\
\text { Études Statistiques } \\
\text { (SCEES) }\end{array}$} & $\begin{array}{l}\text { Enquête Annuelle } \\
\text { Laitière (EAL) }\end{array}$ & $\begin{array}{l}\text { Identification des entreprises laitières par } \\
\text { activité (tables de plusieurs milliers de } \\
\text { lignes) } \\
\text { - Collecte } \\
\text { - Fabrication de produits finis } \\
\text { - Fabrications de produits intermédiaires } \\
\text { - Collecte et Fabrication biologiques } \\
\text { - Fabrication au lait cru }\end{array}$ & 2000 et 2006 \\
\hline & $\begin{array}{l}\text { Recensement } \\
\text { Général Agricole } \\
2000 \text { (RGA) }\end{array}$ & $\begin{array}{l}\text { Données communales : UGB, SAU, } \\
\text { Système agricole dominant }\end{array}$ & Campagne 1999-2000 \\
\hline $\begin{array}{l}\text { Mutualité Sociale } \\
\text { Agricole }\end{array}$ & $\begin{array}{l}\text { Base } \\
\text { administrative de } \\
\text { suivi des cotisants } \\
\text { à la Mutualité } \\
\text { Sociale Agricole } \\
\text { (MSA) }\end{array}$ & $\begin{array}{l}\text { Identification des cotisants à la MSA } \\
\text { présents dans des exploitations laitières. } \\
\text { Sélection d'indicateurs relatifs aux } \\
\text { exploitations laitières }\end{array}$ & $\begin{array}{l}\text { Années civiles } 2004 \text { à } \\
2009\end{array}$ \\
\hline $\begin{array}{l}\text { Ministère de } \\
\text { l'Agriculture }\end{array}$ & $\begin{array}{l}\text { Base Nationale } \\
\text { d'Identification } \\
\text { Bovine (BDNI) }\end{array}$ & $\begin{array}{l}\text { Détentions de bovins (une ligne par animal } \\
\text { et par mouvement) (plusieurs millions de } \\
\text { lignes) } \\
\text { Construction d'une table rassemblant les } \\
\text { données par atelier (exploitations laitières) } \\
\text { (111 } 000 \text { lignes) }\end{array}$ & $\begin{array}{l}\text { Années civiles } 2005 \text { et } \\
2006\end{array}$ \\
\hline $\begin{array}{l}\text { Centre de } \\
\text { Traitement de } \\
\text { I'Information } \\
\text { Génétique (CTIG) }\end{array}$ & $\begin{array}{l}\text { Base du Contrôle } \\
\text { laitier (BCL) }\end{array}$ & $\begin{array}{l}\text { Contrat organisme de contrôle laitier } \\
\text { Lactation } \\
\text { Observation du contrôle laitier }\end{array}$ & $\begin{array}{l}\text { Années civiles } 2005 \text { et } \\
2006\end{array}$ \\
\hline \multirow{2}{*}{$\begin{array}{l}\text { Observatoire du } \\
\text { Développement } \\
\text { Rural (ODR) }\end{array}$} & $\begin{array}{l}\text { Base fournie pour } \\
\text { l'évaluation du } \\
\text { second pilier } \\
\text { (AIDES) }\end{array}$ & $\begin{array}{l}\text { Soutiens directs dans le cadre du second } \\
\text { pilier } \\
\text { Contractualisation de MAE } \\
\text { Identification des exploitations laitières } \\
\text { bénéficiaires. }\end{array}$ & $\begin{array}{l}\text { Années civiles } 2000 \text { à } \\
2010\end{array}$ \\
\hline & Fonds de cartes & $\begin{array}{l}\text { Altitude } \\
\text { Petites Régions Agricoles } \\
\text { Zonage géophysique des Indemnités } \\
\text { Compensatoires de Handicap Naturel } \\
\text { (ICHN) } \\
\text { Zonages AOC }\end{array}$ & \\
\hline $\begin{array}{l}\text { Institut National de } \\
\text { la Statistique et } \\
\text { des Etudes } \\
\text { Economiques } \\
\text { (INSEE) }\end{array}$ & $\begin{array}{l}\text { Recensement de } \\
\text { la population } 1999\end{array}$ & $\begin{array}{l}\text { Densité de population } \\
\text { Zonage en aires urbaines et aires d'emploi } \\
\text { de l'espace rural (Zauer) }\end{array}$ & Année 1999 \\
\hline $\begin{array}{l}\text { Institut National de } \\
\text { l'origine et de la } \\
\text { qualité (INAO) via } \\
\text { le CNIEL }\end{array}$ & $\begin{array}{l}\text { Tableau Excel de } \\
\text { suivi AOP }\end{array}$ & Volumes produits par appellation & $\begin{array}{l}\text { Années civiles } 1980 \text { à } \\
2009\end{array}$ \\
\hline
\end{tabular}




\title{
Résumé
}

La production laitière de montagne rassemble $22 \%$ des producteurs et $15 \%$ des volumes nationaux. Toutefois, les contraintes pédoclimatiques, la pente et l'éloignement engendrent des surcoûts de production et de collecte du lait. Or, la suppression des quotas, et donc la fin de l'ancrage des volumes à l'échelle des départements, exposent plus directement ces systèmes à la concurrence des systèmes laitiers de plaine. Leur pérennité s'en trouve menacée. Un cadre économique d'analyse, multi-niveaux et multi-acteurs, du fonctionnement des marchés laitiers est développé pour analyser la diversité des enjeux d'adaptation post-quotas en montagne. En combinant études de cas et analyse statistique, 43 territoires, regroupés en quatre principaux ensembles sont distingués et cartographiés. Dans les montagnes « à AOP fortes ", la réputation des produits et les dispositifs de coordination spécifiques atténuent l'impact de la libéralisation. Dans les zones « à potentiel AOP», l'enjeu d'adaptation repose sur l'activation des ressources spécifiques et sur leur réappropriation par les éleveurs. Pour les montagnes génériques, les dispositifs de coordination pouvant permettre d'ancrer la production restent à construire. Enfin, dans les zones génériques peu denses, les possibilités de réponse collective sont compromises. L'établissement de nouveaux dispositifs de coordination favorisant la différenciation et la stabilité des marchés demande du temps et des investissements matériels et immatériels conséquents. Il y a donc urgence pour les professionnels et pour l'acteur public à investir dans le développement de capacités d'innovation à l'échelle des territoires.

\begin{abstract}
What outlook for the mountain dairy supply chain after the end of the quota? An approach in terms of competitive system

Mountain dairy farming amounts to $22 \%$ of the producers and $15 \%$ of the national volumes. Due to slope and climate conditions, it is affected by extra milk production and collection costs. With the abolition of the quota system and the end of the production anchorage at the departmental level, mountain dairy farming is increasingly threatened by the competition of plain farming. An economic conceptual framework is developed to analyze the diversity of adjustment issues in mountainous areas. Combining case studies and statistical analysis, 43 areas, gathered in four types, were identified and mapped. In areas with strong PDO cheeses, product reputation and specific coordination instruments reduce the impact of market liberalization. In areas with a potential for PDO cheeses, the issue at stake is the activation of specific resources and their appropriation by the farmers. For areas targeting generic markets, coordination instruments capable of anchoring production are yet to be created. Finally in areas with low farm density, the chances to find collective solutions are small. The establishment of new coordination instruments supporting market segmentation and stabilization takes time and requires investments. The mobilization of economic and public players is urgent.
\end{abstract}

DERVILLÉ M., ALLAIRE G., 2014. Quelles perspectives pour les filières laitières de montagne après la suppression des quotas laitiers ? Une approche en termes de régime de concurrence. In : Spécificités de l'élevage de ruminants en montagne. Grosclaude J., Thibier M., Baumont R. (Eds). Dossier, INRA Prod. Anim., 27, 17-30. 